\title{
Effect of Hund coupling in the one-dimensional SU(4) Hubbard model
}

\author{
Hyun C. Lee, ${ }^{1,2}$ Patrick Azaria, ${ }^{3,4}$ and Edouard Boulat ${ }^{3,5}$ \\ ${ }^{1}$ Department of Physics and Basic Science Research Institute, Sogang University, Seoul, 121-742, Korea \\ ${ }^{2}$ BK21 Physics Research Division and Institute of Basic Science, \\ Department of Physics,Sung Kyun Kwan University, Suwon, 440-746, Korea \\ 3 Laboratoire de Physique Théorique des Liquides, \\ Université Pierre et Marie Curie, 4 Place Jussieu, 75252 Paris, France \\ ${ }^{4}$ CNRS-Centre National de le Recherche Scientifique, 3, rue Michael-Ange, 75016 Paris, France and \\ 5 Department of Physics and Astronomy, Rutgers University, Piscataway, NJ 08855, USA
}

(Dated: October 30, 2018)

\begin{abstract}
The one-dimensional SU(4) Hubbard model perturbed by Hund coupling is studied, away from half-filling, by means of renormalization group and bosonization methods. A spectral gap is always present in the spin-orbital sector irrespective of the magnitude of the Coulomb repulsion. We further distinguish between two qualitatively different regimes. At small Hund coupling, we find that the symmetry of the system is dynamically enlarged to SU(4) at low energy with the result of coherent spin-orbital excitations. When the charge sector is not gapped, a superconducting instability is shown to exist. At large Hund coupling, the symmetry is no longer enlarged to SU(4) and the excitations in the spin sector become incoherent. Furthermore, the superconductivity can be suppressed in favor of the conventional charge density wave state.
\end{abstract}

PACS numbers: 71.10.Hf, 71.10.Pm, 75.10.Jm

\section{INTRODUCTION}

The interplay of spin and orbital degrees of freedom plays an important role in diverse correlated electron systems. $\stackrel{1}{*}$ Recently, the one-dimensional (1D) spinorbital models have been studied intensively motivated by the discovery of the quasi-1D spin-gapped materials, $\mathrm{Na}_{2} \mathrm{Ti}_{2} \mathrm{Sb}_{2} \mathrm{O}$ and $\mathrm{Na}_{2} \mathrm{~V}_{2} \mathrm{O}_{5} \stackrel{2}{2}$ These materials can be modeled by a quarter-filled two-band Hubbard model. Even in this approximation, the situation is rather complex owing to the large number of independent coupling constants in the problem.

A first attempt to understand the effect of the high degeneracy induced by the orbital degrees of freedom was to consider the situation where both orbital and spin degrees of freedom play an identical role. In this case, the two-band Hubbard model possesses a large symmetry and it becomes $\mathrm{SU}(4)$ symmetric in the spin-orbital sector and depends on only one coupling: the Coulomb repulsion $U$. At quarter-filling it was found that spin-orbital and charge degrees of freedom separate at low energy. The spin-orbital sector remains massless for all values of $U>0$ and displays quasi long-range antiferromagnetic order with three critical modes whose dynamics is described by a SU(4) level $1(k=1)$ Wess-Zumino-Witten (WZW) model. This result is in agreement with the exact result by Sutherland for the SU(4) Heisenberg chain ${ }^{3}$. What happens for the $\mathrm{U}(1)$ charge excitations strongly depends on $U$. It was found that a Mott-Hubbard transition from a massless phase at small $U$ to an insulating phase at large $U$ takes place at a nonvanishing critical value $U_{c}=2.8 t \stackrel{4}{\text { Of }}$ of course, the $\mathrm{SU}(4)$ symmetry in the spin-orbital sector is not likely to be present in real materials, so that a systematic study of the effects of possible symmetry breaking operators is necessary to account for the experimental results eventually.

One of the simplest symmetry breaking is to break the $\mathrm{SU}(4)$ symmetry down to $\mathrm{SU}(2)_{\text {spin }} \times \mathrm{SU}(2)_{\text {orbital }}$. A detailed renormalization group study ${ }^{5,6}$ revealed that the $\mathrm{SU}(4)$ symmetry is dynamically enlarged at low energy. Furthermore, the massless phase in the spin-orbital sector survives in an extended region of coupling constant space. These results were confirmed numerically $\underline{\underline{7}}$

In the present work we investigate a different symmetry breaking perturbation which is always present in real materials, the Hund coupling. We also consider the cases of general fillings away from the half-filled one. In comparison with the spin-orbital model studied in Refs. [5] 6], the Hund coupling breaks the SU(4) symmetry further down to $\mathrm{SU}(2)_{\text {spin }} \times \mathrm{U}(1)_{\text {orbital }}$. As for our most important result, we find that a spectral gap opens in the spinorbital sector for an arbitrarily small Hund perturbation. We further distinguish between two qualitatively different regimes. At small Hund coupling, we find that the SU(4) symmetry is dynamically enlarged at low energy like in the spin-orbital model. We further show that a superconducting (SC) instability is present in the charge sector. At large enough Hund perturbation, the SU(4) symmetry is no longer fully enlarged. Instead, we find a partially $\mathrm{SU}(2)$ symmetry enlargement in the orbital sector. In this phase, the SC instability may disappear in favor of the conventional charge density wave (CDW) instability.

This paper is organized as follows. In Sec. II, we present our model and discuss its symmetry properties. The renormalization group analysis at weak coupling is performed in Sec. III, where we also discuss the physical properties in both spin-orbital and charge sector. In Sec. IV, we develop a complementary strong coupling approach for the quarter-filled case. We conclude this paper in Sec. V. 


\section{MODEL HAMILTONIAN AND ITS SYMMETRY PROPERTIES}

The Hamiltonian we consider is the U(4) Hubbard model with a Hund coupling. 8

$$
H=H_{0}+H_{J}
$$

with

$$
\begin{aligned}
H_{0} & =\sum_{i \alpha \alpha^{\prime} \sigma}\left(-t_{i, i+1}^{\alpha \alpha^{\prime}} c_{i \alpha \sigma}^{\dagger} c_{i+1 \alpha^{\prime} \sigma}+\text { H.c. }\right) \\
& +\frac{U}{2} \sum_{i \alpha \alpha^{\prime} \sigma \sigma^{\prime}}\left[n_{i \alpha \sigma} n_{i \alpha^{\prime} \sigma^{\prime}}\left(1-\delta_{\alpha \alpha^{\prime}} \delta_{\sigma \sigma^{\prime}}\right)\right]
\end{aligned}
$$

and

$$
H_{J}=-2 J \sum_{i} \mathbf{S}_{i 1} \cdot \mathbf{S}_{i 2}
$$

where the $c_{i \alpha \sigma}$ are the electron operators at the site $i$ in orbital $\alpha=(1,2)$ with spin $\sigma$. In Eq. (3), $\mathbf{S}_{i \alpha}=$ $\sum_{\sigma \sigma^{\prime}} c_{i \alpha \sigma}^{\dagger}\left[\frac{\sigma}{2}\right]_{\sigma \sigma^{\prime}} c_{i \alpha \sigma^{\prime}}$, denote the spin $1 / 2$ operators of electrons in both bands $\alpha=(1,2)$. We further assume that the hopping is diagonal in orbital space, i.e. $t_{i, i+1}^{\alpha \alpha^{\prime}}=$ $t \delta_{\alpha \alpha^{\prime}}$, and that $U$ and $J$ are positive.

The symmetry properties of Eq. (1) are most clearly seen by introducing the $\mathrm{U}(1)$ charge $Q$ and the $\mathrm{SU}(4)$ spin-orbital generators $\mathcal{T}^{A}, A=(1, \cdots, 15)$, as follows:

$$
\begin{aligned}
Q & =\sum_{i, \alpha \sigma} c_{i \alpha \sigma}^{\dagger} c_{i \alpha \sigma}, \\
\mathcal{T}^{A} & =\sum_{i, \alpha^{\prime} \sigma^{\prime} \alpha \sigma} c_{i, \alpha^{\prime} \sigma^{\prime}}^{\dagger}\left[M^{A}\right]_{\alpha \sigma}^{\alpha^{\prime} \sigma^{\prime}} c_{i, \alpha \sigma},
\end{aligned}
$$

where $M^{A}$ are the generators of SU(4) Lie algebra ${ }^{7.9}$. A convenient explicit realization of the $M^{A}$ is

$$
\frac{1}{\sqrt{2}}\left(\frac{\sigma^{a}}{2}\right)_{\sigma}^{\sigma^{\prime}} \delta_{\alpha}^{\alpha^{\prime}}, \frac{1}{\sqrt{2}} \delta_{\sigma}^{\sigma^{\prime}}\left(\frac{\tau^{a}}{2}\right)_{\alpha}^{\alpha^{\prime}}, \sqrt{2}\left(\frac{\sigma^{a}}{2}\right)_{\sigma}^{\sigma^{\prime}}\left(\frac{\tau^{b}}{2}\right)_{\alpha}^{\alpha^{\prime}}
$$

where $\sigma^{a}$ and $\tau^{a}, a=1,2,3$, are the Pauli matrices acting on spin and orbital space, respectively. An appropriate labeling of the $\mathrm{SU}(4)$ generators in Eq. (5) is as follows. To each SU(4) index $A=(1, \cdots, 15)$ we associate a pair of indices, such that $(a, b) \neq(0,0),(a, b=0,1,2,3)$ with the convention that $\sigma^{0}=\tau^{0}=\operatorname{Id}_{2}$. For an example, the first three generators of Eq. (5) can be alternatively expressed as $M^{(a, 0)}$.

When $J=0$, using Eqs. (45), the Hamiltonian $H$ is clearly seen to commute with both $Q$ and all of $\mathcal{T}^{A}$ 's, thus it is $\mathrm{U}(4)=\mathrm{U}(1)_{\text {charge }} \times \mathrm{SU}(4)_{\text {spin }}$-orbital symmetric.

The Hund coupling $H_{J}$ does not affect the charge sector but breaks the $\mathrm{SU}(4)_{\text {spin-orbital }}$ symmetry. Indeed, when $J \neq 0$, in addition to the obvious $\mathrm{SU}(2)$ invariance in spin space generated by $M^{(a, 0)},(a=1,2,3)$, $H$ is also invariant under the $\mathrm{U}(1)$ orbital group in orbital space generated by $M^{(0,3)}$. Thus, the Hund coupling breaks the $\mathrm{SU}(4)_{\text {spin-orbital }}$ symmetry down to
$\mathrm{SU}(2)_{\text {spin }} \times \mathrm{U}(1)_{\text {orbital }}$. In comparison with the spinorbital model studied in Refs. 5. 6] and Ref. 7], the Hund term breaks the symmetry $\mathrm{SU}(2)_{\text {orbital }}$ further down to $\mathrm{U}(1)_{\text {orbital }}$.

\section{RENORMALIZATION GROUP ANALYSIS AT WEAK COUPLING}

The effective low energy theory associated with the Hamiltonian (1) is obtained by taking the continuum limit in a standard way. At small enough $(U / t, J / t)$ and at low energy, the electron operators $c_{i \alpha \sigma}$ may be expanded near the two Fermi points $\pm k_{F}$ :

$$
c_{i \alpha \sigma}=\sqrt{a_{0}}\left[e^{i k_{F} x} \psi_{R \alpha \sigma}(x)+e^{-i k_{F} x} \psi_{L \alpha \sigma}(x)\right],
$$

where $a_{0}$ is the lattice constant. In the continuum limit, the effective Hamiltonian is expressed naturally in terms of the chiral U(1) and SU(4) current densities: 4

$$
\begin{aligned}
J_{L(R)} & =\sum_{\alpha \sigma} \psi_{L(R), \alpha \sigma}^{\dagger} \psi_{L(R), \alpha \sigma} \\
J_{L(R)}^{\mathcal{A}} & =\sum_{\alpha^{\prime} \sigma^{\prime} \alpha \sigma} \psi_{L(R), \alpha^{\prime} \sigma^{\prime}}^{\dagger}\left[M^{\mathcal{A}}\right]_{\alpha \sigma}^{\alpha^{\prime} \sigma^{\prime}} \psi_{L(R), \alpha \sigma}
\end{aligned}
$$

After some algebra, discarding irrelevant operators, we find that the charge degrees of freedom decouples from the spin-orbital ones away from half-filling:

$$
\mathcal{H}=\mathcal{H}_{c}+\mathcal{H}_{\text {so }}
$$

where

$$
\mathcal{H}_{c}=\int d x\left[\frac{\pi v_{c}}{4}\left(J_{R}^{2}+J_{L}^{2}\right)+g_{c} J_{R} J_{L}\right],
$$

and

$$
\begin{aligned}
\mathcal{H}_{s o} & =\int d x\left\{\frac{2 \pi v_{s o}}{5} \sum_{A}\left(J_{L}^{A} J_{L}^{A}+J_{R}^{A} J_{R}^{A}\right)\right. \\
& -\lambda_{1} \sum_{a}\left[J_{L}^{(a 0)} J_{L}^{(a 0)}+J_{R}^{(a 0)} J_{R}^{(a 0)}\right] \\
& -\lambda_{2} \sum_{a}\left[J_{L}^{(a 3)} J_{L}^{(a 3)}+J_{R}^{(a 3)} J_{R}^{(a 3)}\right] \\
& -\tilde{g}_{1} \sum_{a}\left[J_{L}^{(a 0)} J_{R}^{(a 0)}\right] \\
& -\tilde{g}_{2} \sum_{a}\left[J_{L}^{(a, 1)} J_{R}^{(a, 1)}+J_{L}^{(a, 2)} J_{R}^{(a, 2)}\right] \\
& -\tilde{g}_{3} \sum_{a}\left[J_{L}^{(a 3)} J_{R}^{(a 3)}\right] \\
& -\tilde{g}_{4}\left[J_{L}^{(0,1)} J_{R}^{(0,1)}+J_{L}^{(0,2)} J_{R}^{(0,2)}\right] \\
& \left.-\tilde{g}_{5} J_{L}^{(0,3)} J_{R}^{(0,3)}\right\} .
\end{aligned}
$$


In Eqs. (910), $v_{c}=v_{F}\left(1+3 U a_{0} / 2 \pi v_{F}\right)$ and $v_{s o}=$ $v_{F}\left(1-U a_{0} / 2 \pi v_{F}\right)$ are the charge and spin-orbital velocities, where $v_{F}=2 t a_{0} \sin k_{F} a_{0}$ is the Fermi velocity. We observe that all interactions of Eqs. (910) are marginal and of the current-current type 10 Therefore, the low energy physics will result from a delicate balance among the different interaction terms in Eq. (10). The bare coupling constants in both charge and spin-orbital sectors are expressed in term of $U$ and $J$ as follows:

$$
g_{c}=\frac{3}{4} U a_{0},
$$

and

$$
\begin{array}{ll}
\lambda_{1}=-\lambda_{2}=J a_{0}, & \tilde{g}_{1}=2 U a_{0}+2 J a_{0}, \\
\tilde{g}_{2}=2 U a_{0}+J a_{0}, & \tilde{g}_{3}=2 U a_{0}-2 J a_{0}, \\
\tilde{g}_{4}=2 U a_{0}-3 J a_{0}, & \tilde{g}_{5}=2 U a_{0} .
\end{array}
$$

The effective Hamiltonian in the charge sector [Eq. [9] ] is that of Luttinger liquid:

$$
\mathcal{H}_{c}=\frac{v_{c}}{2} \int d x\left[\frac{1}{K_{c}}\left(\partial_{x} \phi_{c}\right)^{2}+K_{c}\left(\partial_{x} \theta_{c}\right)^{2}\right],
$$

where $\phi_{c}=\phi_{c L}+\phi_{c R}$ and $\theta_{c}=\phi_{c L}-\phi_{c R}$ are the charge boson field and its dual field, respectively. The chiral boson fields $\phi_{c, L / R}$ are defined in terms of currents as follows:

$$
J_{L(R)}(x)=\sqrt{\frac{4}{\pi}} \partial_{x} \phi_{c L(R)}(x) .
$$

Therefore, the charge sector is massless and the low energy properties are determined by the nonuniversal charge exponent $K_{c}$ which is given, at leading order in $U$, by:

$$
K_{c}=\left[1+\frac{3 U a_{0}}{\pi v_{F}}\right]^{-1 / 2}<1 .
$$

The charge velocity can be reexpressed as $v_{c}=v_{F} / K_{c}^{2}$. The situation at hand is similar to what happens in the SU(4) Hubbard model at quarter filling.

The effective Hamiltonian in the spin-orbital sector is that of the $\mathrm{SU}(4)_{1}$ Wess-Zumino-Novikov-Witten (WZWN) model with the central charge $c=3$, perturbed by marginal interactions. This is similar to what happens in the spin-orbital model studied in Refs. [5] 6 ] and Ref. [7]. Due to the complexity of the interaction pattern, namely the five coupling constants instead of three in the spinorbital model, the situation in the spin-orbital sector in the presence of a Hund term requires a careful analysis of the renormalization group (RG) flow. Out of the seven coupling constants entering in Eq. (10), the $\lambda_{1}$ and $\lambda_{2}$ terms are purely chiral and are not renormalized at leading order. Furthermore, they do not influence the scaling of the $\tilde{g}_{i}$ 's. One-loop RG equations are easily found by current algebra method:11.12

$$
\begin{aligned}
& \frac{d g_{1}}{d t}=-g_{1}^{2}-2 g_{2}^{2}-g_{3}^{2}, \\
& \frac{d g_{2}}{d t}=-2 g_{1} g_{2}-g_{2} g_{5}-g_{3} g_{4}, \\
& \frac{d g_{3}}{d t}=-2 g_{1} g_{3}-2 g_{2} g_{4}, \\
& \frac{d g_{4}}{d t}=-3 g_{2} g_{3}-g_{4} g_{5}, \\
& \frac{d g_{5}}{d t}=-3 g_{2}^{2}-g_{4}^{2},
\end{aligned}
$$

where

$$
g_{i}=\frac{\tilde{g}_{i}}{4 \pi v_{s o}}
$$

and $t=\ln L$ is the RG time.

We have performed a detailed numerical analysis of the RG flow associated with Eqs. (16). In the following, we summarize our results.

When $J=0$, the interaction is irrelevant for $U>0$ and all coupling constants flow toward the $\mathrm{SU}(4)_{1}$ fixed point at $g_{i}^{*}=0$. There are no other fixed points associated with Eq. (16). One of our most important results is that a nonzero value of the Hund coupling, $J \neq 0$, destabilizes the $\mathrm{SU}(4)_{1}$ fixed point and drives the system toward strong coupling. This indicates that a gap opens in both spin and orbital sector with $M_{\text {spin }} \sim M_{\text {orbital }} \sim$ $\exp (-C / J)$, where $C$ is a positive constant of order $t$. The present situation is completely different from the one encountered in the spin-orbital model where the critical $\mathrm{SU}(4)_{1}$ phase was not entirely destabilized by the $\mathrm{SU}(2) \times \mathrm{SU}(2)$ symmetry breaking perturbation,$\underline{\underline{5}}$ Though a gap opens in the spectrum, the low energy effective theory still depends on the relative magnitude, $\eta=J / 2 U$, between the Coulomb repulsion and the Hund coupling. Indeed, one finds two qualitatively different behaviors of the RG flow depending on $\eta$.

- The regime A: The $\mathrm{SU}(4)$ symmetric regime. The first is a regime with the enlarged $\mathrm{SU}(4)$ symmetry. This regime occurs for $\eta \ll 1$ or $U \gg J$. This regime will be referred to as the regime A from now on.

With these initial conditions, though all the coupling constants $g_{i}(t) \rightarrow \pm \infty$ when $t \rightarrow t^{*}$, they asymptotically match the following particular RG-invariant ray:

$$
g_{1}=-g_{2}=-g_{3}=g_{4}=g_{5} \rightarrow-\infty .
$$

On that ray, omitting chiral terms, one may write Eq. (10) in an explicit SU(4) invariant form:

$$
\mathcal{H}_{s o}=\sum_{A} \int d x\left[\frac{2 \pi v_{s}^{*}}{5}\left(J_{L}^{A} J_{L}^{A}+J_{R}^{A} J_{R}^{A}\right)-\tilde{g}^{*} J_{L}^{A} J_{R}^{A}\right],
$$

where we have performed the duality transformation for $a, b \neq 0$ :

$$
\begin{aligned}
J_{R}^{a, b} & \rightarrow-J_{R}^{a, b}, \\
J_{L}^{a, b} & \rightarrow J_{L}^{a, b},
\end{aligned}
$$


and $v_{s}^{*}$ is an effective spin-orbital velocity. In fact, there is a velocity anisotropy in the model. We find, however, that to leading order in $J / t$ such an anisotropy of velocities scales to zero at low enough energy 13 Thus, we find that the symmetry is dynamically enlarged to $\mathrm{SU}(4)$ to the one-loop accuracy. Of course the validity this result which relies on the loop expansion may be questioned,, 5.14 .15 but it is reasonable to conjecture that the enlargement of the symmetry is likely to hold beyond the perturbation theory. In any case such an (enlarged) $\mathrm{SU}(4)$ symmetry is meant to be approximate in the sense that small corrections to pure SU(4) behavior should be expected due to the neglected irrelevant operators.

- The regime $B$ : The $\mathrm{SU}(2)_{\text {orbital }}$ enlarged regime. The second is a regime $\mathrm{B}$ where $\mathrm{SU}(2)$ orbital symmetry is partially enlarged [from U(1)]. This regime occurs for the large Hund coupling $J \gg U$ or $\eta \gg 1$. For $\eta \gg 1$ we find that the $\mathrm{SU}(4)$ symmetry is no longer fully enlarged, and instead we observe a partial SU(2) orbital symmetry enlargement in the orbital sector. With the initial conditions satisfying $U \ll J$, the RG flows drive the coupling constants to a regime where:

$$
-\infty \leftarrow g_{4}(t)<g_{5}(t) \ll g_{2}(t)<g_{3}(t) \ll g_{1}(t)<0 .
$$

In this regime the RG equations Eq. (16) can be approximately decoupled. Indeed, at long $\mathrm{RG}$ time, the contributions of $g_{2}$ and $g_{3}$ to the RG equation for $g_{4}$ and $g_{5}$ can be neglected and one obtains:

$$
\begin{aligned}
& \frac{d g_{4}}{d t} \approx-g_{4} g_{5}, \\
& \frac{d g_{5}}{d t} \approx-g_{4}^{2}
\end{aligned}
$$

which are nothing but the RG equations of the $\mathrm{U}(1)$ Thirring model in the orbital sector with effective initial conditions $\left|g_{4}(t)\right|>\left|g_{5}(t)\right|$. In this regime, it is known that the $\mathrm{SU}(2)$ symmetry is restored at larger RG time. Once the anisotropy between $g_{4}$ and $g_{5}$ becomes small, so does the anisotropy between $g_{2}$ and $g_{3}$ as can be seen from the equation

$$
\frac{d\left(g_{2}-g_{3}\right)}{d t} \approx g_{4}\left(g_{2}-g_{3}\right),
$$

since $\left(g_{2}-g_{3}\right)<0$ and $g_{4}<0$. Therefore, in the strong coupling regime, the effective Hamiltonian approximately depends on three independent coupling constants:

$$
\left[g_{4}=g_{5}\right] \ll\left[g_{2}=g_{3}\right] \ll g_{1}<0 .
$$

With the above relation Eq. (24), the interacting part of Eq. (10) displays an $\mathrm{SU}(2)_{\text {spin }} \times \mathrm{SU}(2)_{\text {orbital }}$ symmetry. It is important to notice that no further symmetry restoration is expected since the coupling constant in the orbital sector $g_{4}$ is much larger than the one in the spin sector $g_{1}$. This behavior is in contrast with what happens in the A phase for $\eta \sim 1$.

The study of the physical properties near the boundary between the A and B regimes is a nontrivial problem.
Whether they are separated by a quantum phase transition point or they are smoothly connected by a crossover region can answered only by methods far beyond the perturbation theory. This problem will be addressed elsewhere. Within the one-loop accuracy, we find that the RG flow qualitatively changes from the A-type to the B-type as $\eta$ decreases below $\eta_{0} \sim 0.5$, which is a reasonable value, but we were not able to conclude in favor of a quantum phase transition.

\section{A. Physical Properties and Order Parameters}

\section{Spin-Orbital Sector}

In order to get some insights in the physical properties of the low energy physics it is appropriate to change the parametrizations of the fluctuating fields. We first notice that the two sets of SU $(4)_{1}$ currents, $J_{\frac{R}{L}}^{(a, 0)}$ and $J_{R(L)}^{(0, a)}, a=$ $(1,2,3)$, span two spin and orbital $\mathrm{SU}(2)$ algebras. More precisely, they are $\mathrm{SU}(2)_{k=2}$ currents. This stems from the fact that the $\mathrm{SU}(4)_{k=1} \mathrm{WZW}$ model is equivalent to the sum of two decoupled SU(2) $k=2$ WZW model. ${ }^{16.17}$ As in Ref. [5] we shall take advantage of the representation of the $S U(2)_{k=2}$ algebra in term of three (real) Majorana fermions 17.18 :

$$
\begin{aligned}
J_{R(L)}^{(a, 0)} / \sqrt{2} & =-\frac{i}{2} \epsilon^{a b c} \xi_{s, R(L)}^{b} \xi_{s, R(L)}^{c}, \\
J_{R(L)}^{(0, a)} / \sqrt{2} & =-\frac{i}{2} \epsilon^{a b c} \xi_{t, R(L)}^{b} \xi_{t, R(L)}^{c}, \\
J_{R(L)}^{(a, b)} & =-i \xi_{s, R(L)}^{a} \xi_{t, R(L)}^{b},(a, b) \neq 0,
\end{aligned}
$$

where $\xi_{s, R(L)}^{a}$ and $\xi_{t, R(L)}^{a}, a=(1,2,3)$, are the Majorana fermions associated with the spin and orbital degrees of freedom. In term of these Majorana fermions the effective theories in both $\mathrm{A}$ and $\mathrm{B}$ regimes take a nice form.

In the regime $\mathrm{A}$, the effective low energy Hamiltonian can be obtained from Eq. (10) with the condition $g_{1}=$ $-g_{2}=-g_{3}=g_{4}=g_{5}=g<0$ imposed:

$$
\begin{aligned}
\mathcal{H} & =-i \frac{v_{s}^{*}}{2} \sum_{a}\left[\xi_{s R}^{a} \partial_{x} \xi_{s R}^{a}-\xi_{s L}^{a} \partial_{x} \xi_{s L}^{a}\right] \\
& -i \frac{v_{s}^{*}}{2} \sum_{a}\left[\xi_{t R}^{a} \partial_{x} \xi_{t R}^{a}-\xi_{t L}^{a} \partial_{x} \xi_{t L}^{a}\right] \\
& -g\left[\sum_{a}\left(\kappa_{s}^{a}-\kappa_{t}^{a}\right)\right]^{2},
\end{aligned}
$$

where $\kappa_{s(t)}^{a}=\xi_{s(t) R}^{a} \xi_{s(t) L}^{a}$. The Hamiltonian (26) is easily seen to be $\mathrm{SO}(6)$ invariant upon a duality transformation in the orbital sector:

$$
\xi_{t, R}^{a} \rightarrow-\xi_{t, R}^{a}, \quad \xi_{t, L}^{a} \rightarrow+\xi_{t, L}^{a}
$$

which is the equivalent of Eq. (20) when Eq. (20) is expressed in terms of the Majorana fermions. Under the duality transformation Eq. (27), the Hamiltonian 
(26) becomes that of the integrable $\mathrm{SO}(6)$ Gross-Neveu (GN) mode ${ }^{19.20}$ which has been first obtained in Ref. 5] as the effective low energy theory for the massive phase of the spin-orbital model. In this respect we find that, though the Hund coupling Eq. (3) breaks the original SU(4) symmetry further than spin-orbital model, it is not responsible for the new low energy physics as far as $J$ is not too large in the spin-orbital sector. Therefore, many of the conclusions drawn in Ref. [5 6 6] still hold for moderate values of $J$. In particular, the excitation spectrum consists of a kink and an anti-kink with mass $m$ along with a fundamental fermion of mass $\sqrt{2} m$. The existence of the fundamental fermion as a stable quasiparticle implies that the spin excitations are coherent: a sharp resonance at $\omega=\sqrt{2} m$ is expected to appear in the dynamical structure factor, in particular, the $2 k_{F}$ component of spin-spin correlation function. This can be checked by explicit calculation via order/disorder operator formalism of Ising model [Eqs. [43, 44)].

In the regime $\mathrm{B}$, where $J \gg U$, the effective Hamiltonian is that of two coupled $\mathrm{SO}(3)$ GN models, one in the spin sector and the other in orbital sector:

$$
\begin{aligned}
\mathcal{H}_{s o}= & -i \frac{u_{s}}{2} \sum_{a}\left[\xi_{s R}^{a} \partial_{x} \xi_{s R}^{a}-\xi_{s L}^{a} \partial_{x} \xi_{s L}^{a}\right]-g_{1}\left(\sum_{a} \kappa_{s}^{a}\right)^{2} \\
& -i \frac{u_{t}}{2} \sum_{a}\left[\xi_{t R}^{a} \partial_{x} \xi_{t R}^{a}-\xi_{t L}^{a} \partial_{x} \xi_{t L}^{a}\right]-g_{4}\left(\sum_{a} \kappa_{t}^{a}\right)^{2} \\
& -g_{2}\left(\sum_{a} \kappa_{s}^{a}\right)\left(\sum_{a} \kappa_{t}^{a}\right)
\end{aligned}
$$

which is not integrable in general for arbitrary couplings $\left(g_{1}, g_{2}, g_{4}\right)$. However, in the present case, where $\eta \ll 1$ or $J \gg U$, the effective coupling constants exhibit an interesting hierarchy:

$$
\left|g_{4}\right| \gg\left|g_{2}\right| \gg\left|g_{1}\right|
$$

As a consequence of the above hierarchy, one expects the gap in the orbital sector $\left(\sim e^{-u_{t} /\left|g_{4}\right|}\right)$ to be much larger than any other energy scale in the problem. Therefore a mean-field decoupling of the interaction term in the Hamiltonian (28) is sensible. We can carry out the meanfield decoupling by introducing two Hubbard-Stranovich (HS) fields. For that purpose we rewrite the interactions terms of Eq. (28) as follows:

$$
\begin{aligned}
& g_{1}\left(\sum_{a} \kappa_{s}^{a}\right)^{2}+g_{4}\left(\sum_{a} \kappa_{t}^{a}\right)^{2}+g_{2}\left(\sum_{a} \kappa_{s}^{a}\right)\left(\sum_{a} \kappa_{t}^{a}\right) \\
& =\lambda_{1}\left(\Delta_{t} a_{1}+\Delta_{s} b_{1}\right)^{2}+\lambda_{2}\left(\Delta_{t} a_{2}+\Delta_{s} b_{2}\right)^{2}
\end{aligned}
$$

where the notations are

$$
\begin{aligned}
\Delta_{t} & =-i \sum_{a} \xi_{t R}^{a} \xi_{t L}^{a}, \quad \Delta_{s}=-i \sum_{a} \xi_{s R}^{a} \xi_{s L}^{a}, \\
\lambda_{1} & \sim\left|g_{4}\right|+\frac{g_{2}^{2}}{4\left|g_{4}\right|}, \quad \lambda_{2} \sim 2\left|g_{1}\right|-\frac{g_{2}^{2}}{2\left|g_{4}\right|}, \\
\left(a_{1}, b_{1}\right) & =\left(\frac{\left|g_{4}\right|}{\sqrt{\left(\frac{g_{2}}{2}\right)^{2}+g_{4}^{2}}}, \frac{\left|g_{2}\right| / 2}{\sqrt{\left(\frac{g_{2}}{2}\right)^{2}+g_{4}^{2}}}\right), \\
\left(a_{2}, b_{2}\right) & =\left(-\frac{\left|g_{2}\right| / 2}{\sqrt{\left(\frac{g_{2}}{2}\right)^{2}+g_{4}^{2}}}, \frac{\left|g_{4}\right|}{\sqrt{\left(\frac{g_{2}}{2}\right)^{2}+g_{4}^{2}}}\right) .
\end{aligned}
$$

In Eqs. (31) the hierarchy Eq. (29) was employed to simplify the expressions. Cleary, $\lambda_{1} \gg\left|\lambda_{2}\right|$. Let us assume that $\lambda_{2}$ is positive. Next two HS fields, $\sigma, \zeta$,are introduced to decouple Eq. (30). The resulting Hamiltonian in the action form can be written as

$$
\begin{aligned}
S & =\int d x d \tau\left[+\frac{1}{4 \lambda_{1}} \sigma^{2}+\frac{1}{4 \lambda_{2}} \zeta^{2}\right. \\
& +\frac{1}{2}\left[\xi_{t R}^{a} \xi_{t L}^{a}\right]\left[\begin{array}{cc}
\partial_{\tau}-i u_{t} \partial_{x} & i\left(\sigma a_{1}+\zeta a_{2}\right) \\
-i\left(\sigma a_{1}+\zeta a_{2}\right) & \partial_{\tau}+i u_{t} \partial_{x}
\end{array}\right]\left[\begin{array}{c}
\xi_{t R}^{a} \\
\xi_{t L}^{a}
\end{array}\right] \\
& \left.+\frac{1}{2}\left[\begin{array}{ll}
\xi_{s R}^{a} & \xi_{s L}^{a}
\end{array}\right]\left[\begin{array}{cc}
\partial_{\tau}-i u_{s} \partial_{x} & i\left(\sigma b_{1}+\zeta b_{2}\right) \\
-i\left(\sigma b_{1}+\zeta b_{2}\right) & \partial_{\tau}+i u_{s} \partial_{x}
\end{array}\right]\left[\begin{array}{c}
\xi_{s R}^{a} \\
\xi_{s L}^{a}
\end{array}\right]\right] .
\end{aligned}
$$

Now the Majorana fermions can be integrated out exactly, and the effective action of $\sigma, \zeta$ is obtained. The saddle point approximation ${ }^{21}$ of the effective action of $\sigma, \zeta$ gives

$$
\begin{aligned}
|\langle\sigma\rangle| & \sim \Lambda_{E} e^{-u_{t} /\left|g_{4}\right|}, \\
\langle\zeta\rangle & \sim\langle\sigma\rangle \frac{\left|g_{2}\right|}{2\left|g_{4}\right|}\left(\frac{\lambda_{2}}{\lambda_{1}}\left(\frac{u_{t}}{u_{s}}-1\right)+\frac{3}{\pi} \frac{\lambda_{2}}{u_{s}} \ln \frac{2\left|g_{4}\right|}{\left|g_{2}\right|}\right) .
\end{aligned}
$$

Note that $|\langle\sigma\rangle| \gg|\langle\zeta\rangle|$. The saddle point values of $\Delta_{t}, \Delta_{s}$ are

$$
\begin{aligned}
& \left\langle\Delta_{t}\right\rangle \sim \frac{\langle\sigma\rangle}{2 \lambda_{1}} \\
& \left\langle\Delta_{s}\right\rangle \sim \frac{\left|g_{2}\right|}{2\left|g_{4}\right|} \frac{\langle\sigma\rangle}{2 \lambda_{1}}\left[\frac{u_{t}}{u_{s}}+\frac{3}{\pi} \frac{\lambda_{1}}{u_{s}} \ln \frac{\left|g_{2}\right|}{2\left|g_{4}\right|}\right] .
\end{aligned}
$$

Note that the factor of $\left[\frac{u_{t}}{u_{s}}+\frac{3}{\pi} \frac{\lambda_{1}}{u_{s}} \ln \frac{\left|g_{2}\right|}{2\left|g_{4}\right|}\right]$ in Eq. (35) can be either positive or negative since $\ln \frac{\left|g_{2}\right|}{2\left|g_{4}\right|}<0$. In case the factor is positive we have $\Delta_{s} \Delta_{t}>0$, while the opposite holds for the negative factor.

Both the saddle point value and the fluctuation of $\zeta$ [note the factor $\zeta^{2} / \lambda_{2}$ ] are very small compared to those of $\sigma$. Thus, due to the hierarchy Eq. (29), the HS field $\zeta$ can be neglected in the action Eq. (32). The quantum fluctuations of $\sigma$ are large since $\lambda_{1}$ is large. However, for the spin sector the fluctuations are suppressed by a factor of $b_{1} \sim\left|g_{2}\right| / 2\left|g_{4}\right|$ owing to the hierarchy [see the last line of Eq. [32)]. Thus, we can take the saddle point value of $\sigma$ for the spin sector, while the full quantum fluctuations should be taken into account for the orbital 
sector. Namely, a full integration over $\sigma$ is required for the orbital sector. Then the effective Hamiltonians in both spin and orbital sectors reduce to:

$\mathcal{H}_{\text {spin }}=-i \frac{u_{s}}{2} \sum_{a}\left[\xi_{s R}^{a} \partial_{x} \xi_{s R}^{a}-\xi_{s L}^{a} \partial_{x} \xi_{s L}^{a}\right]-i m_{s}\left(\sum_{a} \kappa_{s}^{a}\right)$,

where $m_{s}=\sigma b_{1} \sim\left|g_{2}\right| \Delta_{t}$, and

$\mathcal{H}_{\text {orbital }}=-i \frac{u_{t}}{2} \sum_{a}\left[\xi_{t R}^{a} \partial_{x} \xi_{t R}^{a}-\xi_{s L}^{a} \partial_{x} \xi_{s L}^{a}\right]-g_{4}\left(\sum_{a} \kappa_{t}^{a}\right)^{2}$.

In this limit, the spin excitations consist of a triplet of free massive Majorana fermions with mass $m_{s}$ (or equivalently off-critical Ising models) that span the spin one representation of $\mathrm{SO}(3)_{\text {spin }}$. This result can be understood qualitatively as follows. Since $J \gg U$, the Hund coupling Eq. (3) dominate in Eq. (1) and the two spin $1 / 2$ operators, $\mathbf{S}_{i 1}$ and $\mathbf{S}_{i 2}$ are effectively bound into a spin one state. Thus, one expects that in this limit the low energy sector of Eq. (II) may be identified with that of a doped spin-one chain ${ }^{22.23}$ This result will be recovered in the next section treating the strong coupling limit. We also expect that in this limit $J \gg U$, all single particle excitations will disappear of the spectrum, since the strong Hund coupling tend to pair electrons. This is indeed the case : the kinks of the $\mathrm{SO}(6) \mathrm{GN}$ model, having the spin-orbital quantum numbers of the electron, vanish and one is left in the lowest energy spectrum with massive Majorana fermions, that is to say magnons. The explicit calculation of (2 $k_{F}$ component of) spin-spin correlation functions using Ising model formalism [ Eqs. [43/44)] shows that there is no sharp resonance. This implies that the spin excitations are incoherent.

The orbital sector itself is described by an $\mathrm{SO}(3)_{\text {orbital }}$ GN model which is integrable. Contrary to what happens in the spin sector, there are no stable (Majorana) fermions in the excitation spectrum ${ }^{20}$. The kink and anti-kink states with mass $m_{t} \sim e^{-u_{t} /\left|g_{4}\right|} \gg m_{s}$ exhaust the excitation spectrum of $\mathrm{SO}(3) \mathrm{GN}$ model.

We see that both $\mathrm{A}$ and $\mathrm{B}$ regimes differ in their spectral properties, which is deeply related to the differences in the underlying symmetries. Starting in the A phase and increasing the value of the Hund coupling $J$ we predict that, above a critical value $J_{c}$, the gap in the orbital sector $m_{t}$ becomes much larger than the spin gap $m_{s}$ : the low energy excitations are exhausted by the spin excitations. Above $J_{c}$ fermionic excitations in the orbital sector disappear and one is left with solely kinks and anti-kinks. This feature is reminiscent of a decoherence phenomenon in orbital-like excitations. This prediction can be tested numerically.

\section{Charge Sector}

The fact that a gap opens in the spin-orbital sector immediately suggests the possibility of charge density wave
(CDW) and superconducting (SC) instabilities. The corresponding order parameters are given by:

$$
\hat{O}_{\mathrm{cdw}}=\sum_{a, \sigma} \psi_{R a \sigma}^{\dagger} \psi_{L a \sigma}+\text { h.c. }
$$

$$
\hat{O}_{\mathrm{sc}, \pm}=\psi_{R 1 \uparrow} \psi_{L 2 \downarrow} \pm \psi_{R 2 \uparrow} \psi_{L 1 \downarrow}+(R \leftrightarrow L),
$$

where $+/$ - stands for singulet/triplet SC.

For the discussion of the correlation functions of the above order parameters it is most convenient to use the Majorana fermion approach. ${ }^{5}$ To this end let us start with the Abelian bosonization formulae:

$$
\psi_{R(L), \alpha \sigma}=\frac{\kappa_{\alpha \sigma}}{\sqrt{2 \pi a_{0}}} e^{ \pm i \sqrt{4 \pi} \phi_{R(L), \alpha \sigma}},
$$

where $\phi_{R(L) \alpha \sigma}$ are boson fields satisfying

$$
\left[\phi_{R, \alpha \sigma}, \phi_{L, \beta \sigma^{\prime}}\right]=i / 4 \delta_{\alpha \beta} \delta_{\sigma, \sigma^{\prime}} .
$$

$\kappa_{\alpha \sigma}$ are the Klein factors which enforce Fermi statistics:

$$
\left\{\kappa_{\alpha \sigma}, \kappa_{\beta \sigma^{\prime}}\right\}=2 \delta_{\alpha \beta} \delta_{\sigma \sigma^{\prime}} .
$$

A convenient choice of basis for the boson fields is

$$
\begin{aligned}
\phi_{c, \frac{R}{L}} & =\frac{1}{2}\left(\phi_{\frac{R}{L}, 1 \uparrow}+\phi_{\frac{R}{L}, 1 \downarrow}+\phi_{\frac{R}{L}, 2 \uparrow}+\phi_{\frac{R}{L}, 2 \downarrow}\right) \\
\phi_{s, \frac{R}{L}} & =\frac{1}{2}\left(\phi_{\frac{R}{L}, 1 \uparrow}-\phi_{\frac{R}{L}, 1 \downarrow}+\phi_{\frac{R}{L}, 2 \uparrow}-\phi_{\frac{R}{L}, 2 \downarrow}\right) \\
\phi_{f, \frac{R}{L}} & =\frac{1}{2}\left(\phi_{\frac{R}{L}, 1 \uparrow}+\phi_{\frac{R}{L}, 1 \downarrow}-\phi_{\frac{R}{L}, 2 \uparrow}-\phi_{\frac{R}{L}, 2 \downarrow}\right) \\
\phi_{s f, \frac{R}{L}} & =\frac{1}{2}\left(\phi_{\frac{R}{L}, 1 \uparrow}-\phi_{\frac{R}{L}, 1 \downarrow}-\phi_{\frac{R}{L}, 2 \uparrow}+\phi_{\frac{R}{L}, 2 \downarrow}\right),
\end{aligned}
$$

Refermionization formulae are given by:

$$
\begin{aligned}
& \left(\frac{\xi^{2}+i \xi^{1}}{\sqrt{2}}\right)_{R(L)}=\frac{\eta_{s}}{\sqrt{2 \pi a_{0}}} e^{ \pm i \sqrt{4 \pi} \phi_{s, R(L)}} \\
& \left(\frac{\xi^{5}+i \xi^{4}}{\sqrt{2}}\right)_{R(L)}=\frac{\eta_{f}}{\sqrt{2 \pi a_{0}}} e^{ \pm i \sqrt{4 \pi} \phi_{f, R(L)}} \\
& \left(\frac{\xi^{6}+i \xi^{3}}{\sqrt{2}}\right)_{R(L)}=\frac{\eta_{s f}}{\sqrt{2 \pi a_{0}}} e^{ \pm i \sqrt{4 \pi} \phi_{s f, R(L)}},
\end{aligned}
$$

where $\eta_{a}$ are new Klein factors. The spin and orbital Majorana fermion triplets are given by $\xi_{s}^{a}=\left(\xi^{1}, \xi^{2}, \xi^{3}\right)$ and $\xi_{t}^{a}=\left(\xi^{4}, \xi^{5}, \xi^{6}\right)$, respectively. In the Majorana fermion basis, both operators Eq. (38) and Eq. (39) are nonlocal, while they take local form in terms of the order $\left(\sigma_{a}\right)$ and the disorder $\left(\mu_{a}\right)$ operators of the six underlying (off-critical) Ising models associated with the six Majorana fermions $\left(\xi_{s}^{a}, \xi_{t}^{a}\right)=\left(\xi^{1}, \ldots, \xi^{6}\right)$. Using the correspondence:

$$
\begin{aligned}
e^{i \sqrt{\pi} \phi_{s}} & \sim \mu_{1} \mu_{2}+i \sigma_{1} \sigma_{2}, \quad e^{i \sqrt{\pi} \theta_{s}} \sim \sigma_{2} \mu_{1}+i \mu_{2} \sigma_{1}, \\
e^{i \sqrt{\pi} \phi_{f}} & \sim \mu_{4} \mu_{5}+i \sigma_{4} \sigma_{5}, \quad e^{i \sqrt{\pi} \theta_{f}} \sim \sigma_{5} \mu_{4}+i \mu_{5} \sigma_{4}, \\
e^{i \sqrt{\pi} \phi_{s f}} & \sim \mu_{3} \mu_{6}+i \sigma_{3} \sigma_{6}, \quad e^{i \sqrt{\pi} \theta_{s f}} \sim \sigma_{6} \mu_{3}+i \mu_{6} \sigma_{3},(44)
\end{aligned}
$$


where $\phi=\phi_{L}+\phi_{R}$ and $\theta=\phi_{L}-\phi_{R}$ we find:

$$
\begin{aligned}
\hat{O}_{\mathrm{cdw}} & \sim \mu_{1} \mu_{2} \mu_{3} \mu_{4} \mu_{5} \mu_{6} \cos \sqrt{\pi} \phi_{c} \\
& +\sigma_{1} \sigma_{2} \sigma_{3} \sigma_{4} \sigma_{5} \sigma_{6} \sin \sqrt{\pi} \phi_{c} .
\end{aligned}
$$

$$
\hat{O}_{\mathrm{sc},+} \sim e^{-i \sqrt{\pi} \theta_{c}}\left[\mu_{1} \mu_{2}+i \sigma_{1} \sigma_{2}\right]\left[\mu_{3} \mu_{4} \mu_{5} \sigma_{6}+\sigma_{3} \sigma_{4} \sigma_{5} \mu_{6}\right]
$$

$$
\hat{O}_{\mathrm{sc},-} \sim e^{-i \sqrt{\pi} \theta_{c}}\left[\mu_{1} \mu_{2}+i \sigma_{1} \sigma_{2}\right]\left[\sigma_{3} \mu_{4} \mu_{5} \mu_{6}-\mu_{3} \sigma_{4} \sigma_{5} \sigma_{6}\right]
$$

We are now in a position to discuss the long distance properties of the correlation functions of the above order parameters.

Consider first the regime A. The spin-orbital dependent parts of Eq. 454647), which has scaling dimension $\frac{3}{4}$, are expressed in terms of products of six order and disorder operators $\sigma_{a}$ and $\mu_{a}$. These $2^{6}$ operators constitute a basis for the primary operators transforming in the spinor representations of $\mathrm{SO}(6)_{1} \mathrm{WZW}$ conformal field theory. Among the spinorial primary operators, there are two $\mathrm{SO}(6)$ singlet primary operators : $\sigma_{1} \sigma_{2} \sigma_{3} \sigma_{4} \sigma_{5} \sigma_{6}$ and $\mu_{1} \mu_{2} \mu_{3} \mu_{4} \mu_{5} \mu_{6}$. At this point it is worth stressing that the enlarged $\mathrm{SO}(6)$ symmetry present at low energy in the the regime $\mathrm{A}$ is different from the original $\mathrm{SO}(6)$ symmetry of the noninteracting theory. Two symmetries are related by the duality transformation Eq. (27) in the orbital sector. Such a transformation interchanges the order and the disorder operators:

$$
\sigma_{a} \leftrightarrow \mu_{a}, a=(4,5,6) .
$$

Consequently only $\hat{O}_{\mathrm{sc},-}$ contains low energy $\mathrm{SO}(6)$ singlets that can take a nonzero average value. Therefore, there exists quasi-long range triplet superconducting order.

$$
\left\langle\hat{O}_{\mathrm{sc},-}^{\dagger}(x, \tau) \hat{O}_{\mathrm{sc},-}(0,0)\right\rangle \sim \frac{1}{\left(x^{2}+u_{c}^{2} \tau^{2}\right)^{1 / 4 K_{c}}},
$$

In contrast to the triplet superconducting order, both CDW and singlet superconductivity have short ranged correlations. A similar analysis can be done when $J<0$ (antiferromagnetic). In this case the model exhibits a singlet superconducting instability rather than a triplet one.

In the regime $\mathrm{B}$ the situation is different. From Eq. (3435) we find that $\Delta_{s} \Delta_{t}$ can be either positive or negative. In case of $\Delta_{s} \Delta_{t}>0$, depending on the sign of $\Delta_{t}$ we have at the mean field level either:

$$
\left\langle\mu_{a=1,2,3}\right\rangle=\left\langle\mu_{a=4,5,6}\right\rangle \neq 0 .
$$

or

$$
\left\langle\sigma_{a=1,2,3}\right\rangle=\left\langle\sigma_{a=4,5,6}\right\rangle \neq 0 .
$$

From Eqs. (45 46477), we conclude that a CDW instability is expected in this case. In case of $\Delta_{s} \Delta_{t}<0$ we have either:

$$
\left\langle\mu_{a=1,2,3}\right\rangle=\left\langle\sigma_{a=4,5,6}\right\rangle \neq 0
$$

or

$$
\left\langle\sigma_{a=1,2,3}\right\rangle=\left\langle\mu_{a=4,5,6}\right\rangle \neq 0
$$

Then a triplet superconductivity is expected in this case.

To summarize, we find that at weak coupling the Hund perturbation always opens a gap in the spin-orbital sector. However, depending on the relative magnitude of $U$ and $J$ we may distinguish between two qualitatively different regimes. At small Hund coupling, $J<U$, the spinorbital sector displays an effective low energy with enlarged SU(4) symmetry and there also exists a triplet superconducting instability in the charge sector. For large Hund coupling, $J \gg U$, there is a partial $\mathrm{SU}(2)_{\text {orbital }}$ restoration, and depending on the relative magnitudes of parameters the superconducting instability may disappear in favor of a CDW quasi-long range order. The way these two regimes are connected is a nontrivial problem and requires a nonperturbative approach.

\section{STRONG COUPLING}

The situation at large coupling depends on the filling as well as the possible umklapp terms $\stackrel{4}{4}$ For incommensurate fillings all umklapp operators are strongly oscillating and may be discarded at low energy. Consequently, the charge excitations are expected to remain massless for all $U$ and $J$. For commensurate filling, i.e. when $n=p / q$, where $p$ and $q$ are co-prime numbers, the possible umklapp operators allowed by parity and translational invariance are of the form:

$$
H_{\text {umklapp }}=\cos \left[4 q \sqrt{\pi} \phi_{c}\right] .
$$

These operators represent the processes that conserve total momentum up to an integer times the Fermi momentum $k_{F}$ in the continuum limit, and they have the scaling dimensions:

$$
\Delta_{q}=4 K_{c} q^{2}
$$

The equation (55) implies that the umklapp operators are irrelevant as far as $K_{c}>1 / 2 q^{2}$. From Eq. (15), we find this is the case as far as $U$ and $J$ are small enough. However, as the coupling constants increase, we expect $K_{c}$ to decrease and possibly to reach the critical value $1 /\left(2 q^{2}\right)$. Below the critical value of $K_{c}$, the umklapp operator Eq. (54) becomes relevant and a gap opens in the charge sector. Thus, the mere existence of a Mott-Hubbard (MU) transition is related to the nonuniversal dependence of $K_{c}$ on the coupling constants $U, J$, and the filling $n$. The dependence is not well-known in general. At present, $K_{c}$ is only known at quarter-filling and for $J=0.4$ In this case, $K_{c}$ reaches its critical value $K_{c}=1 / 2$ at the value $U=2.8 t$, where a MU transition toward an insulating phase has been shown to occur. What happens when $J \neq 0$ and for other commensurate fillings remains an open question. 
In this section we shall focus on the quarter-filled case and make a reasonable hypothesis that a Mott transition still takes place in the presence of a Hund term. Consequently we expect the model described by (1) to be an insulator for large $U$ and $J$ when $n=1$. For this particular filling the strong coupling regime is best achieved by going to the Heisenberg limit. As shown by Arovas and Auerbach 24 all of the relevant low energy states at strong coupling regimes are given by:

$$
\begin{aligned}
& \bar{u}_{0}: \frac{1}{\sqrt{2}}\left(c_{1 \uparrow}^{\dagger} c_{2 \downarrow}^{\dagger}-c_{1 \downarrow}^{\dagger} c_{2 \uparrow}^{\dagger}\right)|0\rangle, \\
& \bar{u}_{1}: c_{1 \uparrow}^{\dagger} c_{2 \uparrow}^{\dagger}|0\rangle, \frac{1}{\sqrt{2}}\left(c_{1 \uparrow}^{\dagger} c_{2 \downarrow}^{\dagger}+c_{1 \downarrow}^{\dagger} c_{2 \uparrow}^{\dagger}\right)|0\rangle, c_{1 \downarrow}^{\dagger} c_{2 \downarrow}^{\dagger}|0\rangle, \\
& \bar{u}_{2}: c_{1 \uparrow}^{\dagger} c_{1 \downarrow}^{\dagger}|0\rangle, c_{2 \uparrow}^{\dagger} c_{12 \downarrow}^{\dagger}|0\rangle .
\end{aligned}
$$

The above states represent inter-orbital spin-singlet, inter-orbital spin-triplet, and intra-orbital spin-singlet states. The energy of each state is given by:

$$
\bar{u}_{0}=U+\frac{3}{2} J, \quad \bar{u}_{1}=U-\frac{1}{2} J, \quad \bar{u}_{2}=U .
$$

The effective strong coupling Hamiltonian depends crucially on the value of $\bar{u}_{1}=U-J / 2$.

\section{A. $\quad U \gg J / 2 \gg t$}

In this case all the energies in Eq. (57) are positive and we can employ the results derived by Arovas and Auerbach ${ }^{24}$ They found that the effective Hamiltonian is given by a generalization of the spin-orbital model:

$$
\begin{aligned}
\mathcal{H}_{\mathrm{eff}} & =\sum_{i}\left[A_{1} \mathbf{S}_{i} \cdot \mathbf{S}_{i+1}+A_{2} \mathbf{T}_{i} \cdot \mathbf{T}_{i+1}+A_{3} T_{i}^{z} T_{i+1}^{z}\right. \\
& \left.+A_{4} \mathbf{S}_{i} \cdot \mathbf{S}_{i+1} \mathbf{T}_{i} \cdot \mathbf{T}_{i+1}+A_{5} T_{i}^{z} T_{i+1}^{z} \mathbf{S}_{i} \cdot \mathbf{S}_{i+1}\right\}_{5}(5)
\end{aligned}
$$

where

$$
\begin{aligned}
& \mathbf{S}_{i}=\sum_{\alpha \sigma \sigma^{\prime}} c_{i \alpha \sigma}^{\dagger}\left[\frac{\boldsymbol{\sigma}}{2}\right]_{\sigma \sigma^{\prime}} c_{i \alpha \sigma^{\prime}} \\
& \mathbf{T}_{i}=\sum_{\sigma \alpha \alpha^{\prime}} c_{i \alpha \sigma}^{\dagger}\left[\frac{\boldsymbol{\sigma}}{2}\right]_{\alpha \alpha^{\prime}} c_{i \alpha^{\prime} \sigma},
\end{aligned}
$$

and

$$
\begin{aligned}
& A_{1}=\frac{2 t^{2}}{U} \frac{\left(1-3 \eta^{2}\right)}{(1-\eta)(1+3 \eta)} \\
& A_{2}=\frac{2 t^{2}}{U} \frac{(1+5 \eta)}{(1-\eta)(1+3 \eta)} \\
& A_{3}=-\frac{2 t^{2}}{U} \frac{3 \eta}{(1+3 \eta)} \\
& A_{4}=\frac{2 t^{2}}{U} \frac{4(1+\eta)}{(1-\eta)(1+3 \eta)} \\
& A_{5}=-4 A_{3}
\end{aligned}
$$

with $\eta \equiv \frac{J}{2 U} \ll 1$. When $\eta=0$, the Hamiltonian Eq. (58) is the $\mathrm{SU}(4)$ invariant antiferromagnetic Heisenberg model studied by Sutherland ${ }^{3}$. Expanding the Hamiltonian Eq. (58) with respect to the $\mathrm{SU}(4)$ symmetric point we obtain

$$
\begin{aligned}
\mathcal{H}_{\mathrm{eff}} & \sim \mathcal{H}_{S U(4)} \\
& +\frac{2 t^{2} \eta}{U} \sum_{i}\left[-2 \mathbf{S}_{i} \cdot \mathbf{S}_{i+1}+3\left(T_{i}^{x} T_{i+1}^{x}+T_{i}^{y} T_{i+1}^{y}\right)\right. \\
& \left.-4 \mathbf{S}_{i} \cdot \mathbf{S}_{i+1} \mathbf{T}_{i} \cdot \mathbf{T}_{i+1}+12 T_{i}^{z} T_{i+1}^{z} \mathbf{S}_{i} \cdot \mathbf{S}_{i+1}\right] \cdot(61)
\end{aligned}
$$

Following Ref. 5] we find that in the continuum limit the effective Hamiltonian Eq. (61) is identified with that of Eq. (10). When $\eta<1$, a RG analysis reveals the same enlargement of $\mathrm{SO}(6)$ symmetry as in the A regime of the weak coupling case. Thus, one may conclude that the $\mathrm{SO}(6)$ symmetric A regime extends from weak to strong couplings for small enough Hund interaction.

\section{B. $t \ll U \ll J / 2$}

In this case $\bar{u}_{1}$ becomes negative and the strong coupling approach developed by Arovas and Auerbach does not apply. However, another strong coupling expansion is sensible when $U \ll J / 2$. Indeed, in this limit the ground state consists of the local spin triplets which contain two electrons per site and spontaneously break translational invariance. Assuming that the spin triplets are located on even sites (such that odd sites are empty) we can find an effective interaction between the local spin triplets. The effective interaction can be determined from the strong coupling expansion to the order of $t^{4}$ in a straightforward way. Let us denote the spin triplet at the $2 j$-th site by $\mathbf{I}_{2 j}$, which is a $S=1$ spin operator. By solving the associated two-site problem up to the order of $t^{4}$, the following effective Hamiltonian can be obtained:

$$
\mathcal{H}_{\mathrm{eff}}^{(4)}=\sum_{j}\left[K_{2}\left(\mathbf{I}_{2 j} \cdot \mathbf{I}_{2 j+2}\right)^{2}+K_{1} \mathbf{I}_{2 j} \cdot \mathbf{I}_{2 j+2}\right],
$$

with

$$
\begin{aligned}
& K_{1}=\frac{8 t^{4}}{5 J^{3}} \frac{7-31 \epsilon / 2+2 \epsilon^{2}+\epsilon^{3} / 2}{(1-\epsilon)^{3}(1-\epsilon / 5)\left(1-\epsilon^{2} / 4\right)} \\
& K_{2}=-\frac{24 t^{4}}{5 J^{3}} \frac{1}{(1-\epsilon / 5)(1-\epsilon)^{2}}
\end{aligned}
$$

where $\epsilon=\frac{2 U}{J} \ll 1$. The Hamiltonian Eq. (62) is that of the antiferromagnetic Heisenberg spin 1 model with exchange $K_{1}$ and a biquadratic coupling $K_{2}<0$. In this regime of coupling constants, the excitations are massive and consist of a triplet of Majorana fermion. ${ }^{25}$ It is however hazardous to conclude that the Haldane magnons constitute the low energy excitations in this strong Hund coupling regime. Indeed, the groundstate for the $t=0, U \ll J$ limit of the model is doubly degenerate for the local spin triplets can be located on even or 
odd sites. In addition to the Haldane magnons of (62) we thus have to take into account the kinks that connect these two groundstates. Note that these kinks carry integer spin, since excitations built out of single electrons have very high energy in this $U \ll J$ regime.

A rough estimate of the energy of kink excitations can be obtained in a static approximation. Consider a pined kink-antikink pair on top of the groundstate of [62), located at sites $p$ and $q$ ( $p, q$ beeing odd integers), that is to say that the local spin triplets are located on even sites $2 i$ for $2 i<p$ and $2 i>q$ and on odd sites $2 i+1$ for $p \leq 2 i+1<q$. When the $t \neq 0$ perturbation is included, this will result in the effective hamiltonian (62) with modified exchange $K_{1,2}$ on the bounds $p$ and $q$. The static approximation is thus equivalent to two bound defects, one weak and one strong (with transparent notations, $K_{1,2}(p) \gg K_{1,2} \gg K_{1,2}(q)$ for the particular configuration we have chosen). The effect of bound defects has been studied in the spin 1 pure Heisenberg chain (without biquadratic exchange), which has the same low energy physics as (62). The defects there lead to the apparition of a triplet excitation inside the Haldane gap 26 It is more than likely that beyond this static approximation, the kinks will form a band of spin 1 excitations once kinetic energy is included, as occurs for holes in the AKLT model 27

While this problem would clearly require a more careful analysis, which goes well beyond the scope of this paper, this simple static picture indicates that the lowest energy excitations will be massive spin 1 kinks. In any case, the low energy spectrum is exhausted by massive magnons. The effective continuum theory in regime $\mathrm{B}$ at weak coupling $t \gg J \gg U$ describes also massive spin 1 particles ; it is thus tempting to postulate a continuity from weak coupling to strong coupling for the low energy excitations.

\section{C. $\quad U \sim J / 2 \gg t$}

In this case the local spin triplet two particle states have very low energy and they will mix with other states with one particle per site in the first order of hopping $t$. These states should be diagonalized first before taking into account the higher order perturbation in $t$. The detailed study of this regime is beyond the scope of this paper.

\section{SUMMARY}

We have studied the one-dimensional SU(4) Hubbard model away from half-filling perturbed by a Hund coupling $J$ in both the weak and strong coupling limits. The Hund coupling turns out to be relevant irrespective of the short ranged Coulomb repulsion $U$ and is responsible for the opening of a spectral gap in the spin-orbital sector. We found two qualitatively different regimes depending on the relative strength between $J$ and $U$.

For small enough Hund coupling $J$, we found that the symmetry is dynamically enlarged to $\mathrm{SU}(4)$ at low energy. In this limit, the the spin-orbital degrees of freedom are described the integrable $\mathrm{SO}(6)$ Gross-Neveu model and the excitations are found to be coherent. In this regime, we found a superconducting instability when the charge excitations are not gapped. For large Hund coupling the SU(4) symmetry is no longer enlarged. Instead, we find that the orbital degrees of freedom decouples at high energy and the low energy excitations lie in the spin sector and consist of the three free massive Majorana fermions. As a result, the spin excitations become incoherent. Our results are summarized in Fig. [1]

\section{Acknowledgments}

P. Azaria and E. Boulat want to acknowledge P. Lecheminant for valuable discussions and suggestions. H.C. Lee is grateful to Prof. K. Ueda for the suggestion of this problem. This work was partially supported by the Korea Science and Engineering Foundation (KOSEF) through the grant No. 1999-2-11400-005-5, and by the Ministry of Education through Brain Korea 21 SNU-SKKU Program.

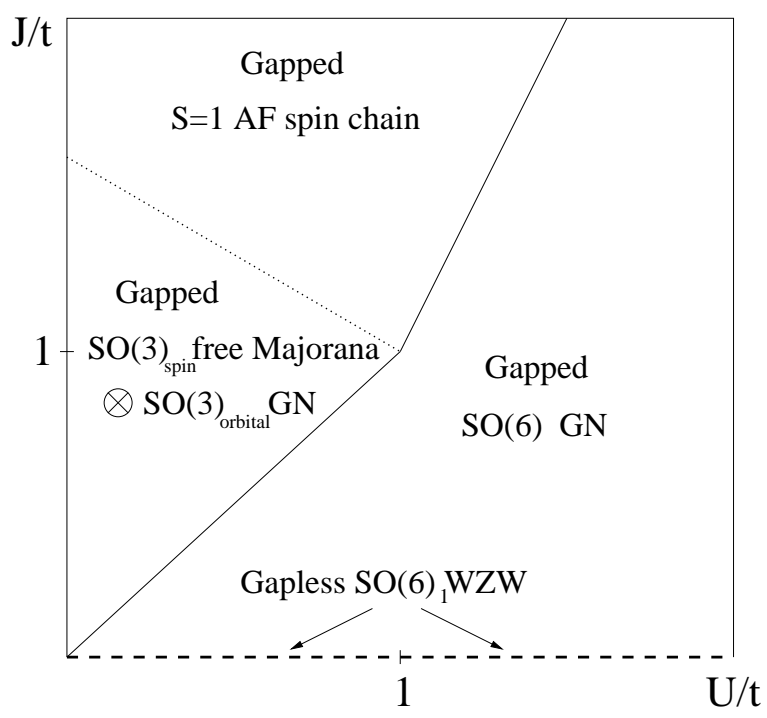

FIG. 1: The phase diagram for the spin-orbital degrees of freedom. The symmetry as well as the effective model at the strong coupling (low energy) regime are indicated. Boundaries represent smooth crossover rather than critical quantum phase transitions. 
1 T. Tokura and N. Nagaosa, Science 288, 462 (2000).

2 E. Axtell el al., J. Solid State Chem. 134, 423 (1997); M. Isobe and Y. Ueda, J. Phys. Soc. Jpn. 65, 1178 (1996).

3 B. Sutherland, Phys. Rev. B 12, 3795 (1975).

4 P. Assaraf, P. Azaria, M. Caffarel, and P. Lecheminant, Phys. Rev. B 60, 2299 (1999).

${ }^{5}$ P. Azaria, A. O. Gogolin, P. Lecheminant, and A. A. Nersesyan, Phys. Rev. Lett. 83, 624 (1999).

${ }^{6}$ P. Azaria, E. Boulat, and P. Lecheminant, Phys. Rev. B 61, 12112 (2000).

7 C. Itoi, S. Qin, and I. Affleck, Phys. Rev. B 61, 6747 (2000).

8 Y. Yamashita, N. Shibata, and K. Ueda, Phys. Rev. B 58, 9114 (1998).

9 From now on the calligraphic letters denote the SU(4) generators.

10 Note the differences in the sign conventions of coupling constants between ours and those of Konik et al. ${ }^{12}$.

11 I. Affleck, in Fields, Strings, and Critical Phenomena, Les Houches 1988, edited by E. Brezin and J. Zinn-Justin (North-Holland, Amsterdam, 1990).

12 R. Konik, H. Saleur, A.W.W. Ludwig, cond-mat/0009166

13 Velocity anisotropy does renormalize at one loop order, provided one performs the perturbative expansion around the non Lorentz invariant fixed describing the free theory with velocity anisotropy. This is best formulated in term of Majorana fields (see Eq. 25), which acquire three different velocities : $v_{s}$ for $\xi_{s}^{a}, v_{t}$ for $\xi_{t}^{4}, \xi_{t}^{5}$ and $v_{6}$ for $\xi_{t}^{6}$. Our one loop calculation shows that in the far infrared, these velocities converge to the one loop $\mathrm{RG}$ invariant $v_{s}^{*}=\left(v_{s}^{3} v_{t}^{2} v_{6}\right)^{1 / 6}$. A very similar situation is discussed in detail in Section III A of Ref. [6].

14 P. Azaria, P. Lecheminant, A. Tsvelik, cond-mat/9806099

15 L. Balents and M. P. A. Fisher, Phys. Rev. B 53, 12133.

16 I. Affleck, Nucl. Phys. B 265, 409 (1985).

17 A. B. Zamolodchikov and V. A. Fateev, Sov. Jour. Part. Nucl. 43, 657 (1986).

18 We follow the notations of P. Azaria et al ${ }^{5,6}$.

19 D. Gross and A. Neveu, Phys. Rev. D 10, 3235 (1974); R. Dashen, B. Hasslacher, and A. Neveu, Phys. Rev. D 12, 2443 (1975).

20 A. B. Zamolodchikov and Al. B. Zamolodchikov, Ann. Phys. (N. Y.) 120, 253 (1979); R. Shankar and E. Witten, Nucl. Phys. B 141, 349 (1978); M. Karowski and H. Thun, Nucl. Phys. B 190, 61 (1981).

21 A. M. Polyakov, Gauge Fields and Strings (Harwood, Chur, Switzerland, 1987).

${ }^{22}$ H. Frahm, M. Pfannmüller, and A. Tsvelik, Phys. Rev. Lett. 81, 2116 (1998).

23 X. Dai, B. Chen, and Z. Su, Phys. Rev. B, 59, 11801 (1999).

24 D. P. Arovas and A. Auerbach, Phys. Rev. B 52, 10114 (1995).

25 D. G. Shelton, A. A. Nersesyan, and A. M. Tsvelik, Phys. Rev. B 53, 8521 (1996)

26 E.S. Sorensen, and I. Affleck, Phys. Rev. B 51, 16115 (1995)

27 S. Zhang, and D.P. Arovas, Phys. Rev. B 40, 2708 (1989) 